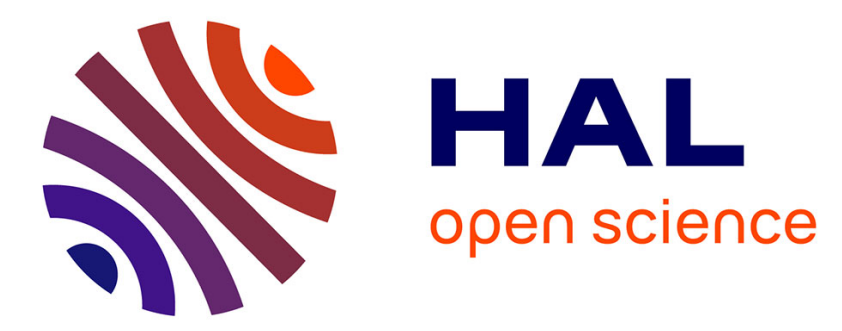

\title{
L'expression morphologique de la personne dans les langues tibéto-birmanes du Nord-Est: une utilisation systématique des techniques possibles
}

François Jacquesson

\section{- To cite this version:}

François Jacquesson. L'expression morphologique de la personne dans les langues tibéto-birmanes du Nord-Est: une utilisation systématique des techniques possibles. Faits de langues, 1997, 5 (10), pp.127-136. 10.3406/flang.1997.1177 . halshs-00008533

\section{HAL Id: halshs-00008533 \\ https://shs.hal.science/halshs-00008533}

Submitted on 13 Dec 2017

HAL is a multi-disciplinary open access archive for the deposit and dissemination of scientific research documents, whether they are published or not. The documents may come from teaching and research institutions in France or abroad, or from public or private research centers.
L'archive ouverte pluridisciplinaire HAL, est destinée au dépôt et à la diffusion de documents scientifiques de niveau recherche, publiés ou non, émanant des établissements d'enseignement et de recherche français ou étrangers, des laboratoires publics ou privés. 


\section{L'expression morphologique de la personne dans les langues} tibéto-birmanes du Nord-Est : une utilisation systématique des techniques possibles

François Jacquesson

\section{Citer ce document / Cite this document :}

Jacquesson François. L'expression morphologique de la personne dans les langues tibéto-birmanes du Nord-Est : une utilisation systématique des techniques possibles. In: Faits de langues, n¹0, Septembre 1997. Les langues d'Asie du Sud. pp. 127-136;

doi : 10.3406/flang.1997.1177

http://www.persee.fr/doc/flang_1244-5460_1997_num_5_10_1177

Document généré le 15/06/2016 


\title{
L'expression morphologique de la personne dans les langues tibéto-birmanes du Nord-Est : une utilisation systématique des techniques possibles
}

\author{
François Jacquesson*
}

à la mémoire de Verrier Elwin

Malgré1 le caractère très imparfait de nos connaissances sur la cinquantaine de langues tibéto-birmanes 2 du nord-est indien, il est clair que la morphosyntaxe de la personne y présente un intérêt considérable. Certaines de ces langues fonctionnent comme le tibetain et le birman, c'est-à-dire que les verbes n'affixent aucune marque personnelle (on sait qu'elles suppléent ce manque morphologique par d'autres moyens, notamment lexicaux), d'autres langues ont au contraire des conjugaisons, parfois compliquées. Cet article donne une idée de quelques cas typiques, après avoir situé la question dans son contexte, et tente de proposer une solution.

Il s'agit donc essentiellement de morphologie comparee, sous l'angle typologique 3 . Le but est de montrer comment une famille de langues, dans sa partie indienne, presente l'éventail complet des possibilités techniques de representation de la personne. Il s'agit, semble-t-il, du seul cas connu où une famille linguistique présente une telle gamme de solutions concretes à un problème universel.

Les langues dont il est question ne sont ni indo-européennes, ni dravidiennes, ni munda ${ }^{4}$. De plus elles sont parentes de nombreuses langues parlées surtout dans les provinces occidentales de la Chine, au Népal, et en Birmanie. Mais il est doublement nécessaire d'en parler dans un ouvrage sur les langues de l'Inde. D'une part certaines

\footnotetext{
* CNRS - Lacito, Courriel : jacquess@vjf.cnrs.fr

1 Lauteur remercie spécialement Miss Chhinjo Meyor et le Pr Gulab Jha de Gauhati.

2 Il n'existe de description moderne, sérieuse et à peu près complète que pour moins d'une dizaine d'entre elles, dont l'angami (Giridhar), le boro (Bhattacharya), le garo (Burling), le mao (Giridhar), le mikir (Gruessner), le tangkhul (Arokianathan); il existe plusieurs bonnes publications sur le meitei. Subbarao devrait donner bientôt une description du mizo. Il existe des données plus ou moins fiables et concises pour une dizaine d'autres; nous avons également utilisé nos enquêtes personnelles (janvier 95, avril 96, octobre 96 à mars 97).

3 La comparaison des formes au sens dune analyse historique n'est pas faite ici. On n'en donnera quiun aperçu à la fin de l'article.

4 Il existe dans le nord-est quelques langues tai, dont les locuteurs sont venus essentiellement du pays Shan de Haute-Birmanie. Ce sont l'ahom, langue éteinte mais qui a eu une grande importance historique, le khamti, l'aiton, le phake, totalisant quelques milliers de locuteurs. II n'en sera pas question ici, ni des dialectes du khasi, qui est une langue non-khmer.
} 
d'entre elles sont anciennement installées en Assam, et ont influencé le parler indoaryen de la vallée du Brahmapoutre, l'assamais. D'autre part beaucoup d'autres, anciennes ou non sur leur terrain actuel, sont parlées par des populations parfois mobiles encore aujourd'hui, et qui donnent au Nord-est indien son profil original.

\section{1 / LES LANGUES TIBÉTO-BIRMANES "ANCIENNES" : PAS D'AFFIXATION PERSONNELLE}

Les Tibéto-Birmans les plus anciens dans la région sont probablement ceux du groupe linguistique Bodo-Garo. Du moins, ce sont les premiers attestés dans les chroniques des souverains Ahom qui ont régné sur l'Assam, et ce sont eux aussi qui vivaient dans la vallée, en royaume constitués ${ }^{5}$, peut-être dès avant l'arrivée des Indoaryens. Les sous-groupes les plus importants sont les Garos du Meghalaya (325000 en 1971 au Meghalaya, 10000 en Assam6), les Boros d'Assam (610 000, en 1971) et les Kokborok du Tripura (300 000); on trouve d'autres sous-groupes Bodo-Garo dans la haute vallée, mais en substance ce sont des gens de vallées et de collines, pas de montagnes.

Burling7 indique qu'en garo, d'une part les verbes ne se conjuguent pas, et d'autre part les pronoms, formellement, ne se différencient guère des noms. Sa catégorie des pronoms personnels repose donc sur un critère sémantique. Pourtant, il existe quelques différences dans le comportement des pronoms. D'abord, beaucoup de pronoms prennent un suffixe $-a: 8$

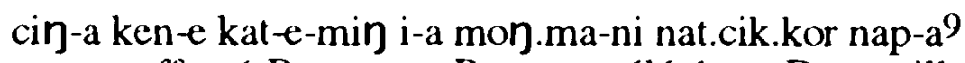

nous-a effrayé-Pt courant-Pt-et ce-a éléphant-Det oreille-trou entrer-Pres

"Effrayés, nous entrâmes en courant dans l'oreille de cet éléphant" 10

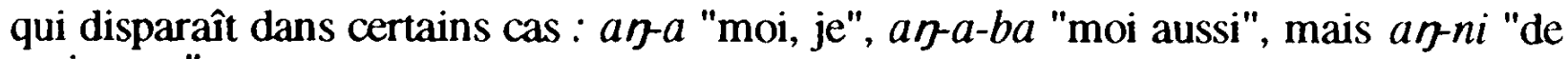
moi, mon".

En boro cependant, le - $a$ est une marque de définitude qui se suffixe aux noms également : kú habbáy "une épine a piqué", et kuwá habbáy (kuwá < kú+a) "l'épine a piqué"11.

5 "The Bodo group [=Bodo-Garo, FJ] includes a widely dispersed group of languages, and seems almost certainly to represent the language that was once spoken generally in the middle and perhaps upper reaches of the Brahmaputra valley." (Burling 1961 : 79).

6 Et d'autres encore au Bangladesh. Le dernier recensement officiel pour l'Assam est de 1971; il existe des statistiques plus récentes (1981) pour certaines provinces voisines. Nous arrondissons les chiffres.

7 Burling 1961.

8 Abréviations : $\mathrm{A}=$ agent d'un verbe transitif, $\mathrm{Ct}=$ continuatif, $\mathrm{DET}=$ déterminant, $\mathrm{O}=$ patient d'un verbe transitif, Pres $=$ présent, $\mathrm{Pt}=$ participe, $\mathrm{U}=$ participant unique d'un verbe intransitif, $\mathrm{POS}=$ possesseur.

9 Dans cet exemples et ceux qui suivent, nous utilisons les tirets pour distinguer dans l'exemple ce qui est distingué dans la glose; et le point pour distinguer ce que le glose traite en amalgame.

10 Burling $1961: 76$.

11 Bhat $1968: 27$. Bhattacharya 1977. 
Il est donc probable que le - $a$ de définitude s'est figé en garo, dans certaines situations partiellement redondantes. Mais le résultat est que les pronoms garo ont développé ainsi une morphologie spéciale qui, malgré tout, en fait formellement une classe particulière. Dans une langue où apparemment, comme en chinois standard du nord, les pronoms sont des noms (comme ils le sont pratiquement en boro), leurs caractéristiques sémantiques, qui motivaient en effet l'emploi, comme dans tant de langues, d'une marque de définitude, ont fini par en faire un trait formel spécifique quand cette marque a disparu ailleurs.

\section{2 / LES LANGUES NAGA DES FRONTIÈRES : SUPPLÉTISME ET FLEXION}

Le terme de Naga, toujours très utilisé et valorisé, recouvre des réalités linguistiques assez différentes. Benedict et Shafer, chacun à sa façon, ont à juste titre séparé les Nagas du nord (ceux du nord du Nagaland et des monts Patkoï) des Nagas du sud, dont la langue est plus proche des parlers kuki, sur quoi nous reviendrons. Bradley, suivant Burling, adopte aussi ce clivage entre deux grands groupes Naga, dont le plus septentrional ferait partie du grand groupe "Sal" 12 .

Toujours est-il que dans la majorité de ces langues, le verbe ne se conjugue pas non plus. Les pronoms personnels ont donc un rôle important. Sur la frontière avec la Birmanie vivent les Khiemnungan ${ }^{13}$, vaste population qui, à cause de sa position géographique, est assez mal connue et dénombrée 14. Dans cette langue, il existe deux pronoms de 1ère personne du singulier ${ }^{15}$. Ni est sujet des verbes intransitifs et patient des transitifs, et $\not \mathfrak{o}$ sert d'agent des transitifs :

\begin{tabular}{l|c|c|c|}
$\begin{array}{l}\text { ni ší ši } \\
\text { moi venir Ct } \\
\text { "je viens" }\end{array}$ & \multicolumn{2}{c}{$\begin{array}{l}\text { nja ni ep ši } \\
\text { toi me voir Ct } \\
\text { "tu me vois" }\end{array}$} & $\begin{array}{l}\text { No nja ep šī } \\
\text { moi toi voir Ct } \\
\text { "je te vois" }\end{array}$ \\
\hline & A & O=U & Pos \\
\hline $1 \mathrm{sg}$. & no & ni & či- \\
\hline $2 \mathrm{sg}$. & nja & nja & a- \\
\hline
\end{tabular}

Cette disparité, qui n'est pas une déclinaison, ne vaut que pour la lère personne, comme on voit. Mais en chang 16 , langue d'un type comparable parlee un peu plus au nord, la différenciation, qui atteint les deux personnes, paraît plus flexionnelle que lexicale. Voici les formes recueillies par Hutton 17 :

12 Voir aussi French 1983, qui est une thèse sur la comparaison lexicale, souvent sur des données très anciennes. Il faut avouer que nous n'en avons guère d'autres.

13 Les renseignements les plus précis sont dans Sardeshpande 1987.

1414000 Khiemnungan au Nagaland en 1971. Cf. Lanusosang 1989. Mais il y en a davantage en Birmanie.

15 Enquête personnelle (avril 96). Nos informateurs sont S. Taniu Lam (surtout), et P. Mono.

1616000 locuteurs en 1971. Lanusosang 1989.

17 Hutton 1987 (1929) : 13. Hutton dit (p.V) sêtre instruit en langue chang à Mokokchung. Mokokchung est la ville principale dune autre tribu, celle des Ao, et cela ne nous renseigne donc pas sur la provenance de ses maîtres. 


\begin{tabular}{|l|c|c|c|}
\hline & $\mathrm{A}$ & $\mathrm{O}=\mathrm{U}$ & $\mathrm{Pos}$ \\
\hline $1 \mathrm{sg}$. & நē & $\eta \eta$ & $\mathrm{ka}-$ \\
\hline $2 \mathrm{sg}$. & nji & nô & $\mathrm{kā}-$ \\
\hline
\end{tabular}

Il semble bien que les formes agent soient issues d'un amalgame avec un suffixe $-i$, qui est le suffixe agent des pronoms et des noms en phom, une autre langue du même ensemble. Nos informateurs 18 disent en effet gai au lieu du $\eta \bar{e}$ de Hutton, qui peut en être une contraction. On ne manquera pas d'observer aussi la parité des finales en $-o$ des autres formes 19 . Enfin, les formes possessives sont encore differentes.

Quoi qu'il en soit de l'histoire des formes, qu'il n'est pas notre propos d'analyser ici, nous avons en khiamnungan un supplétisme limité, et en chang peut-être le résultat d'une déclinaison. Les deux se mêlent en konyak, où les formes sont 20 :

\begin{tabular}{|c|c|c|c|c|}
\hline & A & $\mathrm{O}$ & $\mathrm{U}$ & Pos \\
\hline $1 \mathrm{sg}$. & tawe & $a(\eta)$ & tau & ka-/a- \\
\hline $2 \mathrm{sg}$. & nape & nap & nan & nan- \\
\hline
\end{tabular}

On reconnaît un suffixe agentif en - $e$, mais s'il s'ajoute régulièrement à naク et à tau, la forme $a \eta$, qui est d'ailleurs la forme de 1e sg. en Bodo-Garo, n'appartient pas à cette série. La forme konyak en tau est une innovation, car elle n'existe à peu près que dans cette langue21, mais elle n'a pas occulté cette forme $a \eta$.

Il est clair que les deux phénomènes ont existé. D'une part des affixes indicateurs de l'agent, ou du patient, ou des deux, ont eu tendance à s'amalgamer aux pronoms pour les différencier. Mais cela ne saurait expliquer toutes les formes, et il faut reconnaître que tantôt un nouveau mot (konyak) a été importé dans le système, lexicalement donc; tantôt la divergence des formes entre les différentes fonctions remonte à des faits plus anciens que nous n'apercevons pas bien. Nous voici loin du comportement "isolant" des premiers exemples.

\section{3 / LA CONJUGAISON}

Comme Milewski l'avait indiqué pour les langues d'Amérique du Nord, la personne peut être indiquée par des pronoms (et absente formellement sur le verbe), ou indiquées par un affixe sur le verbe (auquel cas le pronom n'apparaît que dans des conditions particulières). De même que nous voyons une gamme graduée de complexité dans les langues qui n'ont recours qu'aux pronoms, de même dans les langues à conjugaison.

\footnotetext{
18 Yongchingkumla Ongbou Oungh de Tuensang village, et J. Alila Khangshou de Noksen village.

19 Mes informatrices, pour $\mathrm{O}=\mathrm{U}$ disent go ou gwo à la le sg., et $n u$ à la $2 \mathrm{e}$ sg.

20 Informateur: W. Manyeih Tangtok.

21 Pour des vérifications de ce genre, on peut consulter Thurgood, qui, à cause de ses sources, simplifie souvent la question.
} 
Par conjugaison, nous entendons que la personne du sujet est nécessairement exprimée sur le prédicat par une marque formelle. On constatera que dans les langues en question, souvent cette définition ne vaut strictement que pour les $1 \mathrm{e}$ et $2 \mathrm{e}$ personnes.

Enfin, il faut signaler que ce problème a suscité la curiosité depuis plus d'un siècle. Les premiers savants qui étudièrent les langues tibéto-birmanes le firent d'après le tibétain et le birman (d'où le nom de la famille), langues de grande extension qui bénéficient en outre du prestige attaché à une longue tradition écrite. On sait que ces langues n'ont pas de conjugaisons. Lorsqu'on découvrit, d'abord au Népal, quelques langues éparses où il y en avait, l'intérêt typologique de la découverte apparut immédiatement. En effet, on savait bien, au temps où Grierson planifiait le Linguistic Survey of India, que l'Asie du Sud-Est constituait un immense domaine sans conjugaisons, tandis que l'Inde indo-aryenne et dravidienne, de même que les zones iraniennes et même turk plus au nord, présentaient des verbes conjugués. La découverte de langues sporadiques à déclinaison en tibéto-birman donnait un autre sens à l'opposition de ces deux grands blocs aréaux, et l'on se demande encore si ces cas dispersés ne témoignent pas en tibéto-birman d'une ancienne morphologie verbale personnelle. Les découvertes depuis l'époque du Linguistic Survey of India ne cessent de remotiver le débat, car on découvre d'autres langues, parfois des sousgroupes entiers, pourvus de conjugaisons : dans le Nord-est de l'Inde le nocte, le miju et tout récemment le meyor; en Chine par exemple le dulong (=trung) et le gyarong.

\subsection{Variétés de conjugaison dans l'ensemble kuki}

Dans le vaste ensemble des dialectes kuki (appelés "khyin" ou "chin" en Birmanie), on trouve des faits parfois assez simples. En thado par exemple22, le préfixe possessif marque le sujet de l'intransitif; et si le verbe est transitif, il marque l'agent si le patient est "inférieur" dans une hiérarchie $1>2>3$ :

$\begin{array}{lll}\text { a-ma ka-mu-i } & \text { a-ma na-mu-i } & \text { nan ka-mu-i } \\ \text { 3-O 1-voir-Pres } & 3-\mathrm{O} \quad 2 \text {-voir-Pres } & 21 \text {-voir-Pres } \\ \text { "je le vois" } & \text { "tu le vois" } & \text { "je te vois" }\end{array}$

Si l'agent (de la traduction) est "inférieur" au patient, la situation est plus compliquée :

$\begin{array}{llll}\text { nan } & \text { nej-mu-i } & \text { a-man } & \text { na-mu-i } \\ 2 & \text { nej-voir-Pres } & 3-A & 2-\text {-voir-Pres } \\ \text { "tu me vois" } & \text { "il te voit" }\end{array}$

où le nej-ci-dessus est un composé de $n a+i-$, c'est-à-dire du préfixe de $2 \mathrm{e}$ sg. et du préfixe d'exclusif.

En mizo 23 , la grande langue du Mizoram, interviennent à la fois des préfixes complexes comme précédemment, et un suffixe :

22 Notre informateur est Lunsieh Kipgen.

23 Notre informatrice est Lalrinkimi Khawlhring, d'Aizawl. 


$\begin{array}{llll}\text { ka-mhu } & \text { i-mhu } & \text { ka-mhu-ce } & \text { min-mhu } \\ \text { 1-voir } & 2 \text {-voir } & 1 \text {-voir-te } & \text { min-voir } \\ \text { "je (le) vois" } & \text { "tu (le) vois" } & \text { "je te vois" } & \text { "tu me vois" }\end{array}$

Les suffixes apparaissent aussi dans deux autres langues kuki remarquables. Dans un dialecte "chin" de la région de Tiddim 24 , coexistent deux séries d'affixes : une série préfixale dans la langue littéraire, une série suffixale dans la langue ordinaire, mais le fait étrange est que les deux séries sont tout à fait différentes. Or, dans la langue anal25, parlée non loin de là au sud-est du Manipur, nous retrouvons deux séries presque semblables, l'une préfixée et l'autre suffixée :

\begin{tabular}{|c|c|c|}
\cline { 2 - 3 } \multicolumn{1}{c|}{} & \multicolumn{2}{c|}{ tiddim } \\
\cline { 2 - 3 } \multicolumn{1}{c|}{} & littéraire & parlé \\
\hline $1 \mathrm{sg}$. & kă- & - in \\
\hline $2 \mathrm{sg}$. & nă- & $-\mathrm{t} \varepsilon^{?}$ \\
\hline $3 \mathrm{sg}$. & ă- & $-\varnothing$ \\
\hline
\end{tabular}

\begin{tabular}{|c|c|}
\hline \multicolumn{2}{|c|}{ anal } \\
\hline préfixes & suffixes \\
\hline ka- & - ni \\
\hline a- & - ti \\
\hline wa- & $-\varnothing$ \\
\hline
\end{tabular}

mais cette fois les deux séries correspondent à deux classes de verbes, intransitifs et transitifs :

$$
\begin{array}{llll}
\text { avajka.nin } & \text { "je viens" } & \text { ka.tival } & \text { "je (le) vois" } \\
\text { avajka.ti } & \text { "tu viens" } & \text { a.tival } & \text { "tu (le) vois" }
\end{array}
$$

ou coexistent dans les mêmes formes

$$
\text { a.tival.nin "je te vois" ka.tival.ti "tu me vois" }
$$

A y regarder de près, ce système n'est pas si différent de celui du mizo (et de formes d'autres langues non kuki, comme le konyak, que nous ne donnons pas ici).

L'intérêt de cet ensemble kuki est de montrer que, à l'intérieur d'un groupe dialectal homogène, et avec une collection d'affixes personnels fort semblables, on découvre que l'emploi qui en est fait varie de dialecte à dialecte. Une première hypothèse, souvent présentée mais maintenant moins assurée, est que cette variation montre qu'il s'agit d'innovations récentes, différentes d'un dialecte à l'autre. Une autre hypothèse est que le modèle dans l'ensemble kuki est assez complexe, et a été diversement simplifié, ou d'ailleurs compliqué dans certains cas. La discussion reste ouverte tant que nous n'avons pas de données sur davantage de dialectes.

Mais il faut tenir compte de faits "extra-linguistiques" qui sont parfois indûment négligés lorsqu'on ne voit les choses que dans les livres. Les populations kuki-chin vivent de culture semi-itinérante sur brûlis, et les villages sont volontiers déplacés 26. Les influences entre dialectes sont de ce fait d'un type inusité. A la fois, l'innovation

\footnotetext{
24 Henderson 1957.

25 Nos informateurs sont Kevin Pishel et son oncle.

26 Cf. Goswami 1985.
} 
est en effet favorisée, et les structures générales et une partie du lexique sont maintenues à cause de la nécessité de contacts à longue distance (dans le lieu et le temps) entre sous-groupes qui se sont éloignés.

\subsection{Une conjugaison complexe : miju et meyor}

A l'extrémité orientale du nord-est indien vit la tribu des Miju (ou Keman)27. Depuis un siècle peut-être, s'est installé parmi eux un tout petit groupe28 composé de deux clans à l'origine, les Zakhring et les Meyor, qu'on nomme souvent indifféremment Meyor. Ces deux langues, à vrai dire imparfaitement connues ${ }^{29}$, ont en commun une petite partie de leur lexique, et une partie de leur conjugaison. Cette conjugaison est construite d'une façon très différente des précédentes.

Dans ces deux langues, les indices personnels different selon le temps, et il y existe au moins trois temps de cette sorte, ce qui laisse penser qu'il s'agit d'amalgames entre morphème de temps (ou aspect) et indice personnel. Voici pour le miju ${ }^{30}$ le passé et le présent, en laissant le duel de côté; pour un transitif comme $\mathbf{k}^{\text {? }}$ jut "piquer, planter" :

\begin{tabular}{|c|c|c|c|}
\hline passé & 1 & 2 & 3 \\
\hline sg. & $-\mathrm{mi}$ & -măn & -măn \\
\hline pl. & $\tan -$ & -măn.nin & -măn.tau \\
\hline \multicolumn{4}{|l|}{ présent } \\
\hline sg. & -măn & -mun & -mun \\
\hline pl. & -mai & $-\mathrm{me}$ & -mun.tau \\
\hline
\end{tabular}

Pour un verbe intransitif comme tai "venir", les formes sont identiques au passé, mais un peu différentes au présent :

\begin{tabular}{|c|c|c|c|}
\hline présent & 1 & 2 & 3 \\
\hline sg. & $-\mathrm{mi}$ & -măj & -măn \\
\hline pl. & \multicolumn{3}{|c|}{-nt ${ }^{3}$ it aux 3 personnes } \\
\hline
\end{tabular}

On voit qu'en effet un morphème "auxiliaire" -m- intervient souvent.

En meyor, et c'est ce qui fait son interêt particulier, les faits sont encore plus complexes. Il est indispensable d'en avoir une idée pour en parler un peu. D'abord le cas du verbe intransitif, par exemple avec kuk "venir", qui est suivi de :

273600 personnes en 1981 .

28250 personnes d'après S. Barua in Dutta \& Ahmad 1995 : 406. Sans doute un peu plus en réalité.

29 Pour le miju-keman: Das Gupta 1977, Sun Hongkai 1980. La connaissance du meyor repose entièrement sur mon informatrice Chhinjo Meyor (janvier 95, avril 96).

30 Sun Hongkai 1980. 


\begin{tabular}{|c|c|c|c|}
\hline passé & 1 & 2 & 3 \\
\hline sg. & lin & čilo & lo \\
\hline pl. & laj & čilonaj & lokaj \\
\hline \multicolumn{4}{|l|}{ présent } \\
\hline sg. & $\min$ & 'i & $\eta$ \\
\hline pl. & maj & 'ini & n \\
\hline \multicolumn{4}{|l|}{ futur } \\
\hline sg. & $\min$ & 'ilek & lek \\
\hline pl. & maj & 'ilekmaj & lekko \\
\hline
\end{tabular}

les formes transitives, avec un verbe comme pak "porter (un animé) sur son dos", utilisent au passé des formes particulières, mais les mêmes au présent :

\begin{tabular}{|c|c|c|}
\hline passsé & présent & \\
\hline jo? & $\min$ & "je te" ou "je le" \\
\hline ču & i & "tu me" \\
\hline $\begin{array}{l}\text { čik } \\
\text { phu }\end{array}$ & & "tu le" \\
\hline
\end{tabular}

On constate qu'au présent, l'indice marque A comme il marque U; mais qu'au passé, la situation est plus compliquée.

On trouverait des faits du même ordre par exemple en trung (dulung), une langue parlée non loin de là, à la frontière commune de l'Inde, de la Chine, et de la Birmanie.

Il est possible, mais inutile ici, d'analyser ces formes pour montrer comment elles sont constituées, comment le morphème auxiliaire change selon le "temps" et parfois selon la personne (comme en anglais classique d'ailleurs, où shall et will alternent selon la personne, pour des raisons qui sont sémantiques à l'origine).

Ce qui est important ici, c'est de voir que le degré d'élaboration de la "conjugaison" peut être très poussé, à la fois du point de vue des constituants morphologiques qui entrent en jeu, et du point de vue des amalgames qui finissent par constituer une véritable flexion selon le "temps" et la "personne".

\section{4 / CONCLUSION}

Dans un espace géographique relativement restreint, nous constatons une variété inégalée de types formels. Ou bien les verbes restent insensibles à la personne, et ce sont des pronoms qui la marquent. On a alors tous les cas possibles : ou les pronoms ne marquent que la personne, ou ils agrègent des suffixes casuels, ou ils présentent des cas de supplétisme selon la fonction, surtout pour la première personne. Ou encore, ce sont les formes verbales qui changent selon la personne d'un ou, parfois, de deux actants. Alors, tantôt ces affixes personnels s'associent clairement au thème verbal en fonction de la personne impliquée, tantôt plusieurs séries d'affixes entrent en jeu, tantôt la situation se complique encore en fonction du "temps". 
Pourtant, il est indéniable que toutes ces langues sont tibéto-birmanes, et parmi elles certaines sont étroitement liées. C'est un cas exceptionnel de diversité morphosyntaxique, qui permet de montrer l'étendue continue des possibilités, et donc de tenter de les classer.

On peut certes opposer typologiquement les langues qui utilisent des pronoms et celles qui utilisent des affixes (et qui ont également des pronoms, bien entendu).

Mais on s'aperçoit que les deux "types" communiquent. Dans les langues kuki par exemple, lorsque deux peronnes en fonction différente sont en jeu, l'une est représentée par un pronom, l'autre par un préfixe (ordinairement identique au préfixe "possessif"). Ce qui différencie les langues, c'est l'assignation de chaque série à chaque fonction. En thado, n'apparaissent comme pronom que les personnes "inférieures", donc seulement 2 et 3, quelle que soit leur fonction, tandis que 1 "supérieur" ne peut être marqué que par un préfixe. Si 1 et 2 sont en cause, la distinction est plus subtile : $1>2$ donne selon la règle précédente 1 comme préfixe et 2 comme pronom, tandis que $2>1$ est le cas unique où intervient un composé de deux préfixes, dont l'exclusif. En mizo, les pronoms peuvent être également utilisés avec les préfixes, mais $1>2$ et $2>1$ entraînent tous deux des solutions particulières : comme en thado, $2>1$ est traité par un préfixe composé, et $1>2$ donne un agent préfixé accompagné d'un pronom spécial postposé. En anal, une série pronominale, devenue suffixée, complète de même une série préfixée, avec cette singularité que le pronom qui, lorsqu'il est seul, marque le participant unique d'un intransitif, marque l'agent si la forme est d'autre part préfixée. En meyor, où les amalgames avec les auxiliaires rendent l'analyse plus délicate, nous percevons aussi, curieusement, des formes préfixées et suffixées: au passé de l'intransitif par exemple, la le pers. lin (l+in), où l'indice personnel suit l'auxiliaire, s'oppose à la 2e pers. älo (čitlo), où l'indice précedde l'auxiliaire, de sorte que nous retrouvons dans l'alternance de position des deux personnes à la fois l'opposition de personnes qu'on constate dans le transitif kuki, et l'alternance de position (préfixe / pronom) qu'on vient de rappeler.

En somme, sans qu'on puisse encore dresser un tableau exhaustif des possibilités, ni s'assurer du jeu complet entre personnes et fonctions, on voit cependant assez bien que ce sont les implications fonctionnelles propres à chacune des deux premières personnes, la le vers l'agent, la 2 e vers le patient, qui permettent d'entrevoir une rationalité commune aux nombreuses morphosyntaxes existantes, et dont nous avons présenté quelques-unes.

Voici donc un vaste ensemble de langues où un principe sémantique simple semble être à l'origine de morphosyntaxes d'une diversité sans exemple ailleurs. C'est donc que le principe fut et est assez puissant pour orienter, sous des formulations extrêmement diverses, des langues pourtant réunies aussi par lui. 


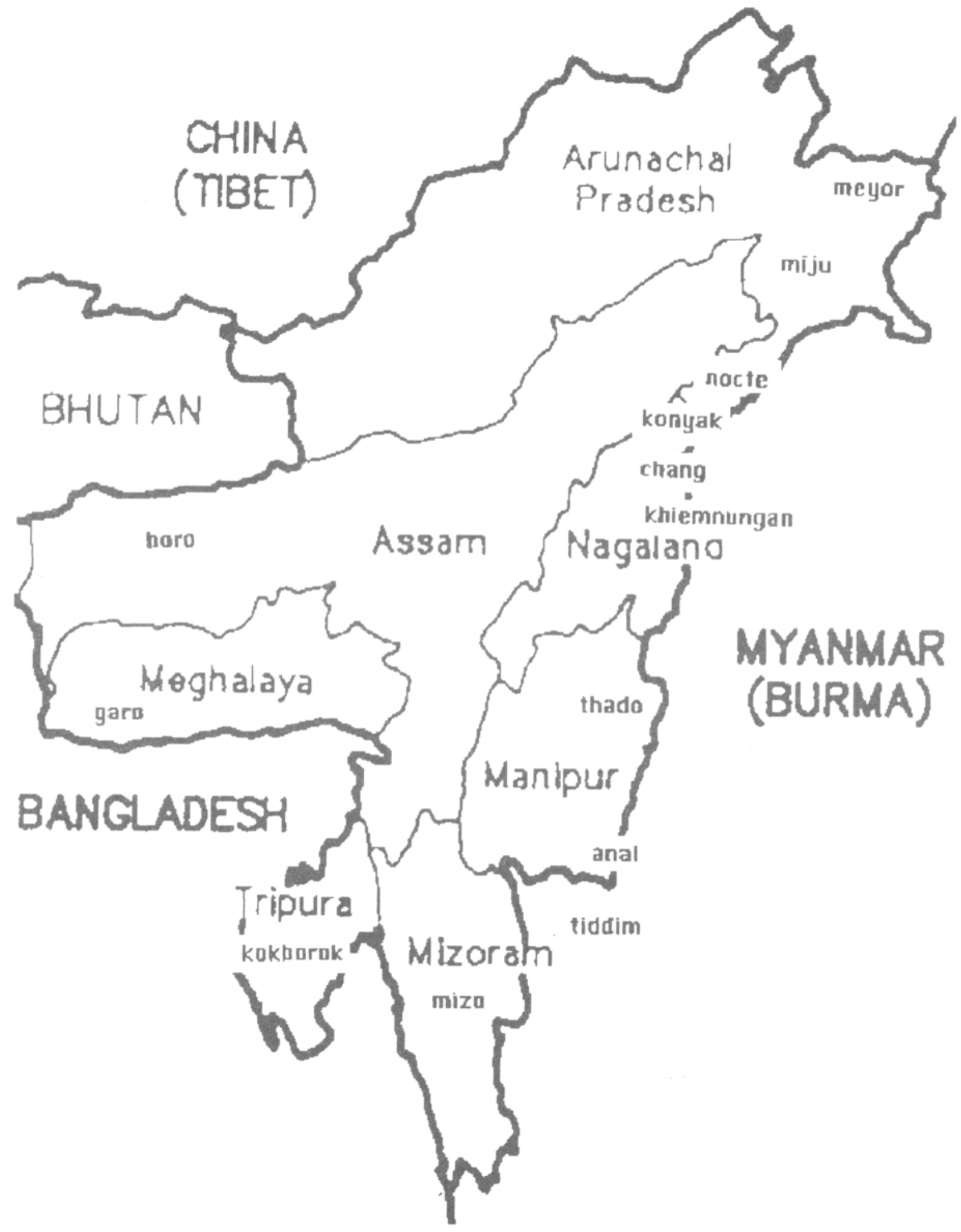

